\title{
A Multihop Clustering Algorithm for Energy Saving in Wireless Sensor Networks
}

\author{
Saeed Ebadi \\ Department of Computer, Islamic Azad University, Tabriz Branch, Tabriz, Iran \\ Correspondence should be addressed to Saeed Ebadi, sa_ebadi@yahoo.com \\ Received 27 January 2012; Accepted 5 March 2012 \\ Academic Editors: A. Alomainy and C. S. Hong
}

Copyright () 2012 Saeed Ebadi. This is an open access article distributed under the Creative Commons Attribution License, which permits unrestricted use, distribution, and reproduction in any medium, provided the original work is properly cited.

One of the important problems for wireless sensor networks is increasing the network lifetime. Clustering is an efficient technique for prolonging the lifetime of wireless sensor networks. This papers propose a multihop clustering algorithm (MHC) for energy saving in wireless sensor networks. MHC selects the clusterheads according to the two parameters the remaining energy and node degree. Also cluster heads select their members according to the two parameters of sensor the remaining energy and the distance to its cluster head. MHC is done in three phases quickly. Simulation results show that the proposed algorithm increases the network lifetime more than 16 percent compared of the LEACH protocol.

\section{Introduction}

Along recent advances in microsensors, VLSI technologies, and wireless communication fields, another kind of wireless network named wireless sensor networks (WSN) has been appeared. The sensor networks can be used for various application areas (e.g., health, military, and home) [1]. For different application areas, there are different technical issues that researchers are currently resolving. A sensor network is composed of a large number of sensor nodes that are densely deployed either inside the phenomenon or very close to it [1].

They are usually made up with wireless sensor nodes, small, cheap, and resource-limited devices sensing the environment and Communicating with each other [2]. Wireless distributed microsensor systems will enable the reliable monitoring of a variety of environments for both civil and military applications. For example, for a security system, acoustic, seismic, and video sensors can be used to form an ad hoc network to detect intrusions. Microsensors can also be used to monitor machines for fault detection and diagnosis. communication between the sensor nodes and the base station is expensive, and there are no high-energy nodes through which communication can proceed. Reliable environment monitoring is important in a variety of commercial and military applications [3].
These networks have many limits in the network lifetime such as processing power, memory, and transmitter power. Energy conservation has been identified as the key challenge in the design and operation of these networks. Dense positioning of sensor nodes can improve network connectivity and fault tolerance; the main drawback of such deployment is obvious: data collected by neighboring nodes is highly spatially corelated. Hence, if the raw sensor readings are to be sent to the sink, considerable amount of redundant data would have to be communicated through the network. Clearly, transmission of redundant data implies waste of valuable node energy [4]. The essential operation in clustering is to select a set of cluster heads from the set of nodes in the network. Cluster heads are responsible for coordination among the nodes within their clusters and aggregation of their data (intra-cluster coordination) and communication with each other and/or with external observers on behalf of their clusters (inter-cluster communication) [5].

In clustering, all sensors in network are divided into clusters each of which has a sensor as cluster head $(\mathrm{CH})$. These sensors are homogenous and have same capability of processing, memory, and power consumption. The Function of cluster head is cluster management, data collection from cluster members, and sending them to the base station (BS). In recent years, multiple algorithms have been presented for 
clustering in wireless sensor network. in Section 2, we describe LEACH [3], LNCA [4], HEED [5], and other protocols [6-9].

\section{Related Works}

LEACH protocol [3] is one of the known protocols in the clustering field. Each sensor decides whether to become a cluster head for the current round or not. This decision is based on the recommended percentage of cluster heads for the network and number of times the sensor has been a cluster head so far. Each sensor chooses a random number between 0 and 1 . If the number is less than a threshold, the sensor becomes a cluster head for the current round.

LNCA [4] (local negotiated clustering algorithm) presents a novel clustering algorithm, which employs the similarity of nodes readings as an important criterion in cluster formation. LNCA greatly reduces the data-reporting-related traffic with reasonable clustering cost. HEED [5] periodically selects cluster heads according to a hybrid of the sensor residual energy and a secondary parameter, such as node proximity to its neighbors or node degree.

HEED sets an initial percentage of cluster heads among all $n$ nodes $\left(C_{\text {prob }}\right)$, assuming that an optimal percentage cannot be computed a priori. $C_{\text {prob }}$ is only used to limit the initial cluster head announcements and has no direct impact on the final clusters. Before a node starts executing HEED, it sets its probability of becoming a cluster head, $\mathrm{CH}_{\text {prob }}$, as [5]:

$$
\mathrm{CH}_{\text {prob }}=C_{\text {prob }} \times \frac{E_{\text {residual }}}{E_{\max }},
$$

where $E_{\text {residual }}$ is the estimated current residual energy in the node and $E_{\max }$ is a reference maximum energy, which is typically identical for all nodes. In EE_SPEED [8], clustering routing is based on a weight function, which is a combination of the three factors: delay, energy and speed. MCBT [9] proposes a distributed algorithm to create a stable backbone by selecting the nodes with higher energy or degree as the cluster heads.

\section{MHC Algorithm}

3.1. Assumptions. MHC algorithm presented the following assumptions. set of $n$ sensors are spread in environments nonuniformly. Considering that clustering is multihop, density of sensors near base station is higher. All sensors have the same capability. The Base station and sensors location are fixed. Each sensor has a unique ID. Communication between sensors and their cluster heads is single hop and communication between cluster heads and BS is multihop.

For transmiting $l$-bit data to distance $d$, consuming energy is calculated as [3]

$$
E_{T x}(l, d)= \begin{cases}l \times E_{\text {elect }}+l \times \varepsilon_{\mathrm{fs}} \times d^{2}, & d \leq d_{o} \\ l \times E_{\text {elect }}+l \times \varepsilon_{\mathrm{mp}} \times d^{4}, & d>d_{o} .\end{cases}
$$

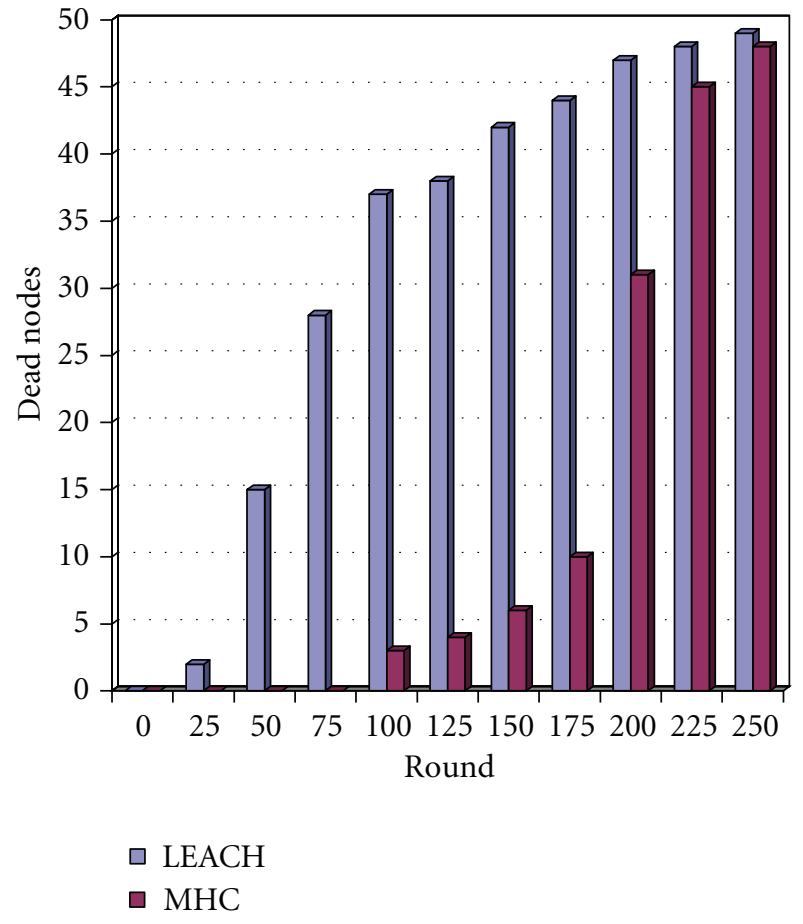

Figure 1: Number of dead nodes.

In here $d_{o}$ threshold is calculated as in:

$$
d_{o} \equiv \sqrt{\frac{\varepsilon_{\mathrm{fs}}}{\varepsilon_{\mathrm{mp}}}} .
$$

Also consuming energy to receive $k$-bits data is calculated as $[3,5]$

$$
E_{R x}(l)=l \times E_{\text {elect }} .
$$

3.2. Proposed Algorithm. In this paper, a multihop clustering algorithm (MHC) is proposed for energy saving in wireless sensor networks. In MHC, the sensor is selected as cluster head according to the two parameters remaining energy and node degree. Also cluster heads select their members according to the two parameters of sensor the remaining energy and the distance to its cluster head.

MHC is done in three phases, initial, hierarchical, and final phases. This algorithm performs the initial phase only in the beginning of network clustering and the final phase after finishing network clustering. However, the algorithm repeats the hierarchical phase from the first level until last level hierarchically finishs the clustering of the entire network. In data collection phase, sensors compare gathered environmental data with its neighboring data. If data was similar [4], the sensor stores ID of message sender in the list of its neighbors and counts the number of neighboring and sets it to $N_{i}$ variable.

In initial phase at beginning of clustering, BS that as a cluster head of first level, transmit a "Start" message in transition range of sensors, and tell starting of clustering to all. Only sensors that are close to BS, receive this message. 


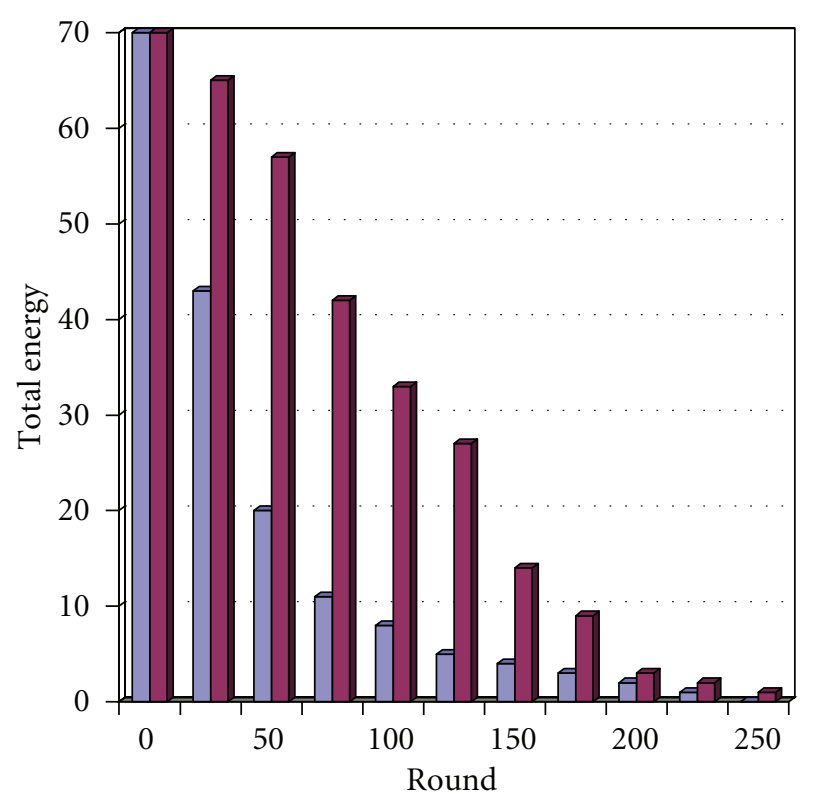

$\square \mathrm{LEACH}$

$\square \mathrm{MHC}$

Figure 2: Total Energy of nodes in Network.

TABLE 1: Simulation parameters.

\begin{tabular}{ll}
\hline Parameter & Value \\
\hline Field & $300 * 300$ \\
BS location & $(0,150)$ \\
Numbers of sensors $(n)$ & 50 \\
Sensor range & $150 \mathrm{~m}$ \\
$E_{r}$ & $1.5 \mathrm{~J}$ \\
$E_{\text {elec }}$ & $50 \mathrm{~nJ}$ \\
$E_{\text {amp }}$ & $100 \mathrm{pJ}$ \\
$E_{\mathrm{DA}}$ & $5 \mathrm{~nJ}$ \\
Package length & $1000 \mathrm{bit}$ \\
\hline
\end{tabular}

Hierarchical phase is done in four steps hierarchically that whole sensors of network can clustered.

Step 1. In this step, the sensors that received "Start" massage (whether from BS at first or other sensors) compute the value of $\mathrm{T}$ for themselves. the value of $\mathrm{T}$ is calculated based on two factors of sensor remaining energy $\left(E_{r}\right)$ and number of neighbors $\left(N_{a}\right)$ as:

$$
\mathrm{T}=\mathrm{E}_{r}+N_{a} .
$$

After the computing value of $\mathrm{T}$, sensors send it to their neighbors. Sensors receive the value of $\mathrm{T}$ from neighbors and compare it with their $\mathrm{T}$ value. the sensor that has largest $\mathrm{T}$ value selects itself as a cluster head and selects sender of "Start" message as its high-level cluster head.

Step 2. After MHC selects cluster heads, cluster heads must select its members. In this step, cluster heads send a "join to me" message to all nodes in its range. Every sensor node that receive this messages from cluster heads, calculate value of $\mathrm{T}_{\mathrm{CH}}$ for itself. The Value of $\mathrm{T}_{\mathrm{CH}}$ is calculated based on two factors of sensor remaining energy $\left(E_{r}\right)$ and the distance to its cluster head $\left(S_{r}\right)$ as

$$
\mathrm{T}_{\mathrm{CH}}=E_{r}+S_{r} .
$$

After $\mathrm{T}_{\mathrm{CH}}$ computing, sensor selects the sender of the highest $\mathrm{T}_{\mathrm{CH}}$ as its cluster head and send a "join to you" message to it.

Step 3. In this step, cluster heads create a TDMA scheduler according to cluster members that receive "join to you" message from them and send TDMA to all.

Step 4. In this step, cluster heads transmit "Start" massage in itself radio range for selecting clusters of next level and Step 1 repeated hierarchically that entire network be clustering.

In the final phase data are collected by sensors from environment and delivered to BS by cluster heads. This work performed by members and cluster heads is to be followed.

Member sensors gather data from environment and send to itself cluster head in its scheduled transmission time. The radios transmitter of other sensors is turned off until their allocated transmission time to save the energy.

Cluster heads receive data packets from its members and also receive from low levels cluster heads. Then they perform data aggregation on the received data and transmit to its high level cluster head.

\section{Performance Evaluation}

We simulate MHC and LEACH protocols by MATLAB software and compare their efficiency of them. The proposed algorithm is simulated in $300 * 300$ field. Simulation results are averaged after 20 times running. The parameters of simulation are considered as in Table 1 . In Table $1, E_{\mathrm{DA}}$ is required energy for data aggregation.

Figure 1 shows the number of dead sensors because of finished batteries during the rounds in MHC and LEACH protocols. Comparing the two graphs of Figure 1 implies that the network lifetime of MHC will be increases further compared to LEACH protocol.

This variation was created because MHC is multihop versus LEACH. When half the nodes of network die, almost efficiency of network is low. In mean state, MHC improve this time more than four times. Also, it improves the dead time of last node (e.g., network life time) 16\%.

Figure 2 shows total energy of nodes in network during the rounds in MHC and LEACH protocols.

Figure 1 that indicates the energy consumption of nodes in LEACH is more than that in MHC.

\section{Conclusions}

For extending the lifetime of wireless sensor networks, in this paper an energy efficient cluster-based routing algorithm is proposed to increase life-time of wireless sensor networks. 
MHC selects the cluster heads according to the two parameters the remaining energy and node degree. MHC is done in three phases quickly. Also the members join the cluster heads according to two factors, the maximum energy and the distance to cluster head. Then, we simulate MHC and LEACH protocols and compare their efficiency. Simulation results show that $\mathrm{MHC}$ prolongs lifetime of networks $16 \%$ by nearly. MHC has a good scalability and network lifetime prolongs further with increasing scale of network.

\section{References}

[1] I. F. Akyildiz, W. Su, Y. Sankarasubramaniam, and E. Cayirci, "A survey on sensor networks," IEEE Communications Magazine, vol. 40, no. 8, pp. 102-105, 2002.

[2] H. A. Tanaka, H. Nakao, and K. Shinohara, "Self-organizing timing allocation mechanism in distributed wireless sensor networks," IEICE Electronics Express, vol. 6, no. 22, pp. 15621568, 2009.

[3] W. R. Heinzelman, A. Chandrakasan, and H. Balakrishnan, "Energy-efficient communication protocol for wireless microsensor networks," in Proceedings of the 33rd Annual Hawaii International Conference on System Siences (HICSS-33), p. 223, January 2000.

[4] D. Xia and N. Vlajic, "Near-optimal node clustering in wireless sensor networks for environment monitoring," in Proceedings of the 21st International Conference on Advanced Information Networking and Applications (AINA '07), pp. 632-641, May 2007.

[5] O. Younis and S. Fahmy, "HEED: a hybrid, energy-efficient, distributed clustering approach for ad hoc sensor networks," IEEE Transactions on Mobile Computing, vol. 3, no. 4, pp. 366379, 2004.

[6] H. S. Lee, K. T. Kim, and H. Y. Youn, "A new cluster head selection scheme for long lifetime of wireless sensor networks," in Computational Science and Its Applications (ICCSA 2006), vol. 3983 of Lecture Notes in Computer Science, pp. 519-528, 2006.

[7] D. Shangwei and Y. Xiaobu, "Exploring hierarchy architecture for wireless sensor networks management," in Proceedings of the IFIP International Conference on Wireless and Optical Communications Networks, p. 6, April 2006.

[8] M. S. Kordafshari, A. Pourkabirian, K. Faez, and A. M. Rahimabadi, "Energy-efficient speed routing protocol for wireless sensor networks," Proceedings of the 5th Advanced International Conference on Telecommunications (AICT '09), pp. 267-271, 2009.

[9] I. Shin, M. Kim, M. W. Mutka, H. Choo, and T.-J. Lee, "MCBT: multi-Hop cluster based stable backbone trees for data collection and dissemination in WSNs," MDPI Sensors, vol. 9, no. 8, pp. 6028-6045, 2009. 

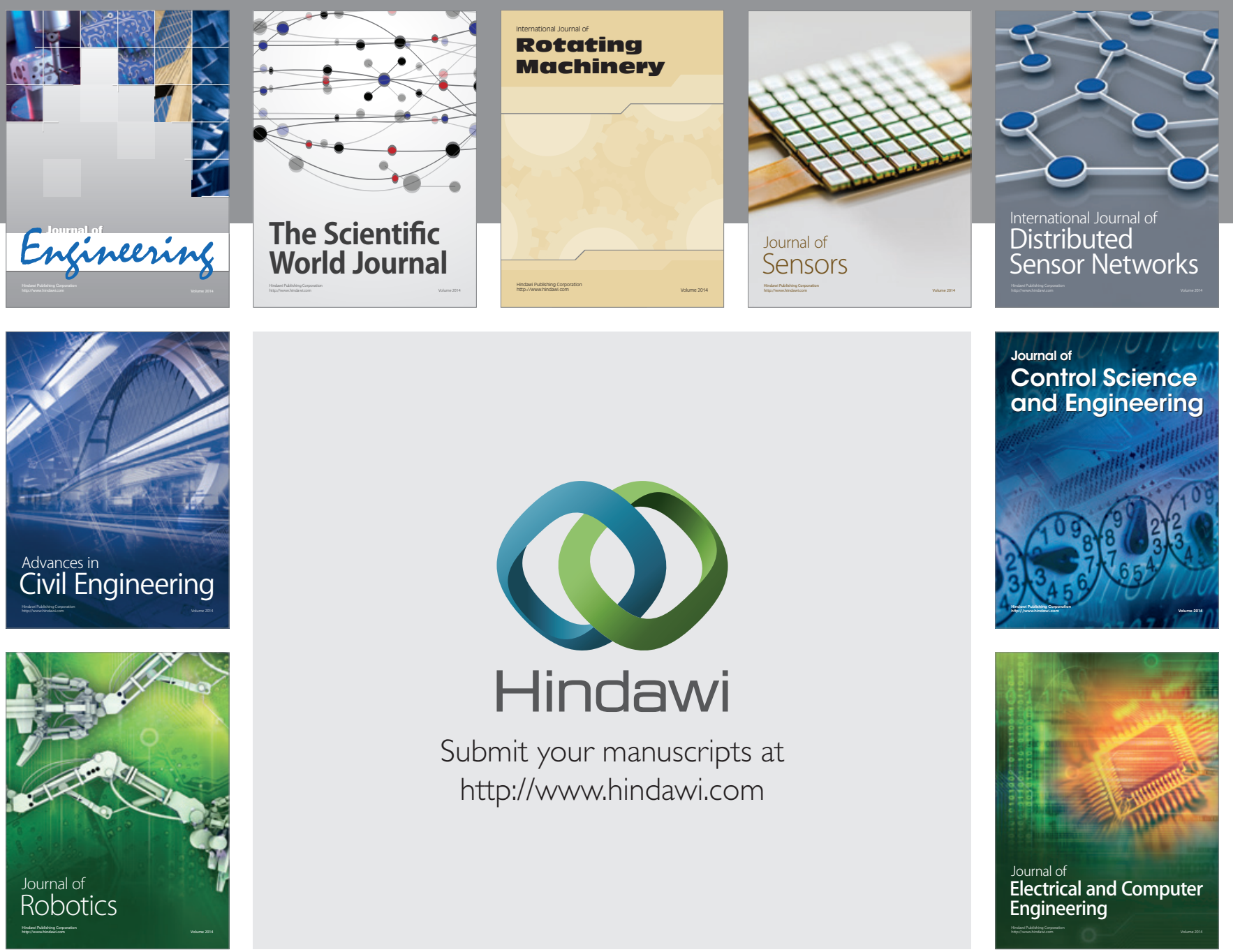

Submit your manuscripts at

http://www.hindawi.com
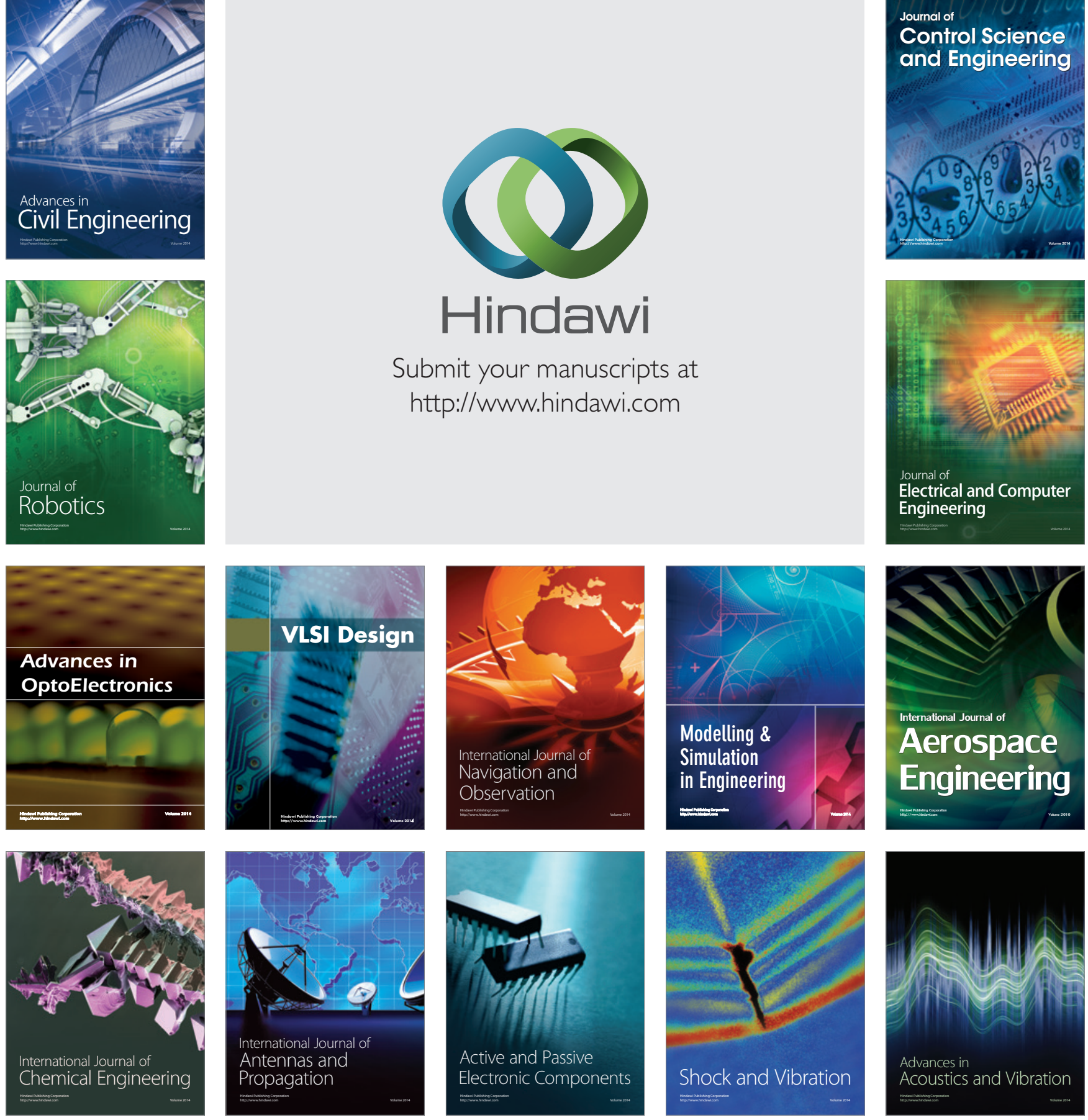